behaviour as a result of experience. It is a prerequisite for episodic memory, which is selectively impaired in clinical amnesia. Primate models of amnesia are valid only if monkeys possess the capacity for secondary mental representation and only if the tests employed depend critically on its use. It is not self-evident that the conventional test of amnesia in monkeys (impaired performance on delayed nonmatching to sample tasks) does depend on secondary mental representation. Secondary mental representation is also necessary for the comparison of alternatives within one cognitive manoeuvre. Monkeys with hippocampal dysfunction are impaired on conditional learning when trials are presented in an order which invites comparison of alternatives, but are not impaired on the same task when the order of trials artificially segregates component parts of the task. The hippocampus may be particularly important in the process of secondary mental representation, and it is this contribution to cognition which may underlie the critical involvement of the hippocampus in episodic memory.

\title{
Symposium 6: Memory and amnesia in man and animals
}

Organized by D. Gaffan

\section{Symp 6/1}

\section{MAPPING FUNCTIONAL SYSTEMS IN THE RAT BRAIN TO HELP IDENTIFY PATHWAYS FOR MEMORY}

\section{J.P. Aggleton}

School of Psychology, Cardiff University, Cardiff, CF1 3YG Wales, UK

Rather than seek to model amnesia directly in rats, we have adopted the approach of analysing component aspects of memory and uncovering the neural systems supporting these various elements. Particular attention has focussed on functional relationships with the hippocampus as this structure is critical for normal episodic memory in humans. Two techniques have been used to uncover functional relationships among regions that might play a key role in memory. Lesion studies have highlighted the many similarities between the effects of damage to the fornix, anterior thalamic nuclei, and hippocampus on tests of spatial memory; similarities that have been supported by recent disconnection studies involving contralateral lesions of the anterior thalamus and fornix. In contrast, regions such as the perirhinal cortex and the medial dorsal thalamic nucleus show quite different lesion induced deficits that can be dissociated from those of the hippocampus. Mapping studies using the expression of the immediate early gene c-fos support these distinctions. Perihinal cortex shows increased gene activity when a rat is shown novel visual items, while the hippocampus remains unchanged. In contrast, the hippocampus and anterior thalamic nuclei, but not the perirhinal cortex, show enhanced fos production after performing allocentric spatial memory tasks. Importantly, there are changes in c-fos activation in both the hippocampus and perirhinal cortex when the spatial configuration of familiar items is changed. Such findings not only highlight the importance of the anterior thalamic nuclei for hippocampal function, but also reveal a range of interactions both within and between distinct systems. 


\section{Symp 6/2}

\section{NEURAL MECHANISMS FOR TOP-DOWN SELECTION OF HIGH-LEVEL VISUAL REPRESENTATIONS: A COMMON ARCHITECTURE FOR ATTENTION AND MEMORY RETRIEVAL?}

\section{Chelazzi}

Department of Neurological and Vision Sciences, Section of Physiology, University of Verona, Verona, Italy

In the primate brain visual information from the retina is processed through a hierarchy of cortical visual areas. A number of single-unit recording studies in the awake behaving macaque have shown that activity at several, perhaps all, stages of this hierarchy is profoundly modulated by selective attention. In particular, attentional signals allow representations of stimuli of interest to proceed through the hierarchy and eventually gain access to working memory and motor control, while representations of other, competing stimuli are suppressed. Suppression may be stronger at later stages of the visual cortical processing stream, such as the inferotemporal and posterior parietal cortices, but modest effects have also been detected at the level of primary visual cortex. It has been proposed that attention-related modulation along the occipito-temporal (ventral) pathway of cortical visual processing depends on top-down control signals specifying the representations that are relevant in a given behavioral context. In particular, control signals may give supremacy to the representation of relevant inputs at the expense of irrelevant ones. One candidate source of attentional control signals is lateral prefrontal cortex, an area known to play a key role in many cognitive functions, including working memory. The theory holds that the content of working memory controls which inputs from the retina are given full processing through the cortical visual system and are eventually given access to conscious experience and motor control. A possible route through which prefrontal cortex may determine attentional modulation of visual processing is through its connections with regions of the inferotemporal cortex. Many lines of evidence have also shown that the macaque inferotemporal cortex is likely to be the storehouse of visual long-term memories. The storage process itself depends critically on the limbic system but, once the consolidation of memories is complete, the neocortex in the temporal lobe appears to be the main deposit of long-term visual memories. While the neocortex in the temporal lobe may be sufficient for the long-term storage of visual memories, other structures seem to be necessary for their active retrieval. Notably, research from the past few years has shown that signals from prefrontal cortex may be essential for the retrieval of mnemonic representations stored in the temporal lobe. It is thus conceivable that the same set of projections from prefrontal cortex onto inferotemporal cortex are critical for two distinct cognitive functions: the context-dependent attentional selection of information coming from the retina and the retrieval of visual long-term memories. A possible scenario is that these two functions are indeed two expressions of the same underlying neuronal operation, namely the selective activation of high-level visual representations. In one case prefrontal signals would select from among the multiple central representations elicited by the retinal input. In the other case the same signals would select from among the countless quiescent representations deposited as long-term memories. 


\section{Symp 6/3}

\section{A CHOLINERGIC EXPLANATION OF MEDIAL TEMPORAL AMNESIA \\ D. Gaffan \\ Department of Experimental Psychology, Oxford University, UK}

The traditional explanation of dense amnesia after medial temporal lesions is that amnesia is caused by damage to the hippocampus and related structures. This view has been falsified by accumulated evidence in man and animals showing that selective damage to these structures produces a mild impairment of episodic memory which does not amount to dense amnesia. An alternative view is that dense amnesia after medial temporal lesions is caused by interruption of afferents to the temporal cortex from the basal forebrain. These afferents travel to the temporal cortex through three pathways, namely the anterior temporal stem, the amygdala and the fornix-fimbria, and all of these pathways are damaged in dense medial temporal amnesia. In four experiments using different memory tasks, we tested the effects on memory of sectioning some or all of these three pathways in macaque monkeys. In a test of scenespecific memory for objects, which is analogous in some ways to human episodic memory, section of fornix alone or section of amygdala and temporal stem sparing the fornix, each produced a significant but mild impairment. When fornix section was added to the section of temporal stem and amygdala in this tasks, however, a very severe impairment resulted. In an object recognition memory task (delayed matching-to-sample), a severe impairment was seen after section of temporal stem and amygdala alone, with and without fornix section; this impairment was significantly more severe than that which was seen in the same task after amygdalectomy leaving the temporal stem intact, with or without fornix section. Animals with combined section of the temporal stem, amygdala and fornix were also impaired in object reward associations learning. However, the retention of pre-operatively acquired object-reward associations was at high level. These results show that the pattern of impairments after section of temporal stem, amygdala and fornix in the monkey, leaving the hippocampus intact, resembles human dense amnesia and is different from the effects of hippocampal lesions in the monkey which do not resemble human dense amnesia. Furthermore, an impairment of equal severity in object-reward association learning was demonstrated in a separate group of animals which received crossed unilateral lesions of the medial forebrain bundle in one hemisphere and of inferior temporal cortex in the other hemisphere, and in animals with selective cholinergic lesions of the basal forebrain. Thus, medial temporal lesions in dense amnesia have their effect on memory by disrupting cholinergic projections from the basal forebrain to the temporal cortex. 


\section{Symp 6/4 \\ ROLE OF PERIRHINAL CORTEX IN OBJECT INFORMATION PROCESSING AND OBJECT IDENTIFICATION}

E. A. Murray

Laboratory of Neuropsychology, National Institute of Mental Health, Bethesda, MD, USA

The perirhinal cortex, a strip of cortex located in the ventromedial temporal lobe, plays a critical role in visual recognition memory. In addition, perirhinal cortex has been found to be important for relating together the different sensory features of objects, thereby facilitating object identification. Recent work suggests that perirhinal cortex contributes to both perception and memory. In "perception", it serves as the final stage in a ventral visual cortical processing stream, known as the "what" pathway, that is devoted to the perception and identification of environmental stimuli. Its special contribution to this type of processing is held to be in the representation of complex conjunctions of features. In "memory", perirhinal cortex participates in acquisition, retrieval, and long-term storage. Evidence from ablation and physiological studies suggests a critical role for the primate perirhinal cortex in the formation and retrieval of both intramodal and crossmodal stimulus-stimulus associations, associations that presumably endow objects with meaning. The perirhinal cortex, together with other cortical fields, also serves as a site of long-term storage of such knowledge. Its central role in many associative processes is likely due to its pivotal anatomical position linking representations stored in diverse sensory and motor areas, as opposed to any special computational function it might possess.

Symposium 7: From sensation to perception: Extra-retinal influences in parietal cortex Organized by U. Ilg

\section{Symp 7/1}

\section{NON-RETINOCENTRIC REPRESENTATION OF VISUAL INFORMATION IN MONKEY POSTERIOR PARIETAL CORTEX}

\section{F. Bremmer}

Allg. Zoologie und Neurobiologie, Ruhr-Universitaet Bochum, D-44780 Bochum, Germany

A fundamental problem in the control of visually guided movement consists in the different frames of reference in which sensory and motor signals do occur. The incoming sensory signals are organized in a retinotopic manner throughout several stages of the visual system whereas the outgoing motor commands are encoded with respect to singular muscles. Considering a typical interaction with an environment like reaching out for a target, obviously the problem arises in how to relate the incoming sensory signal with the required motor output. One hypothesis for solving this problem is a so-called coordinate transformation from retinocentric signals to signals encoded in a cranio-, ego- or even allocentric frame of reference. Several studies on awake monkeys have shown that activity of neurons in many cortical 

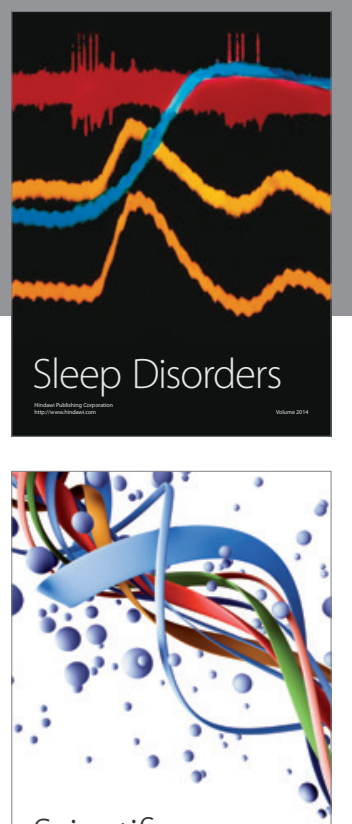

Scientifica
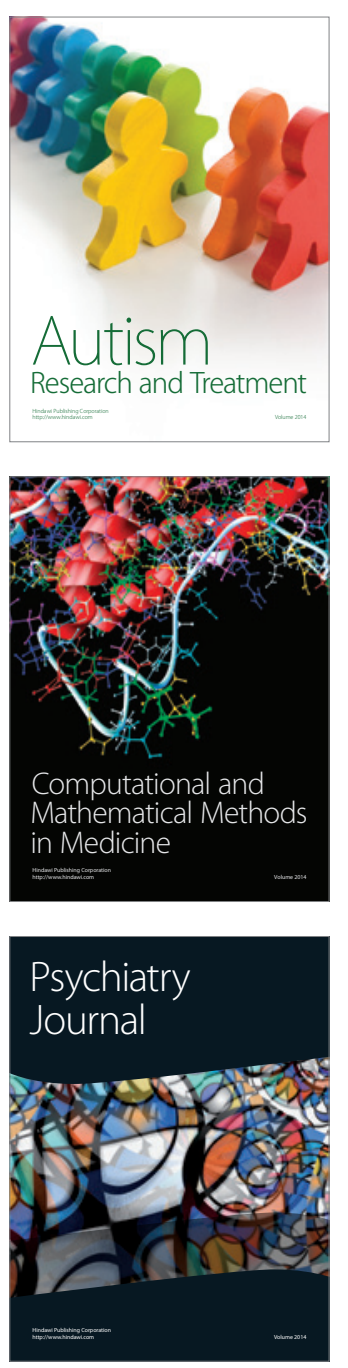
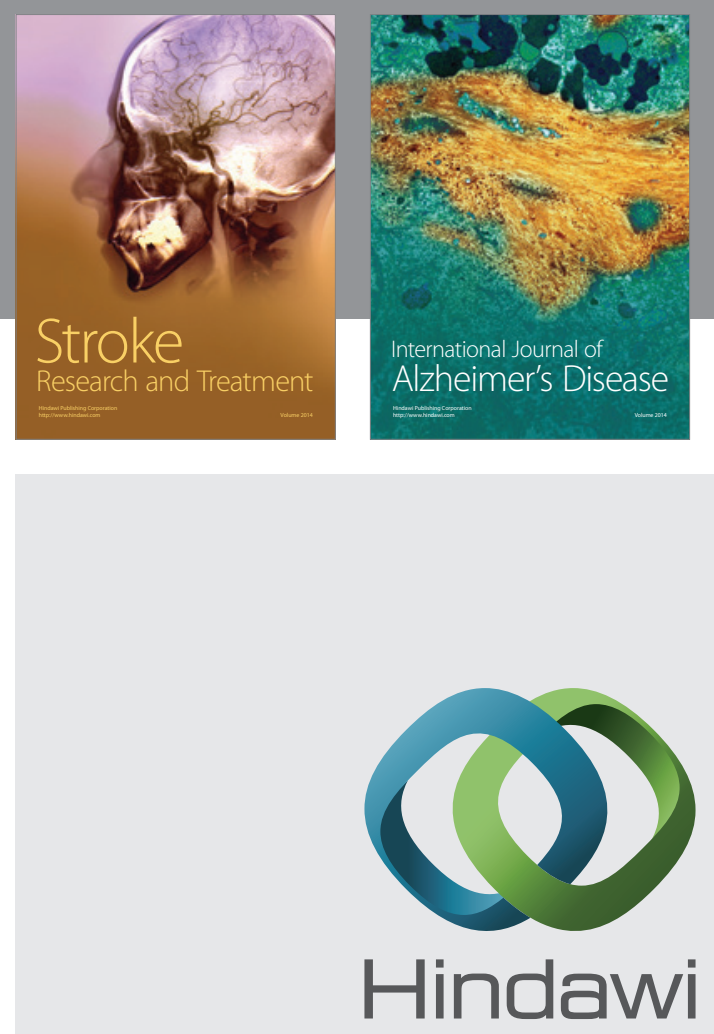

Submit your manuscripts at

http://www.hindawi.com
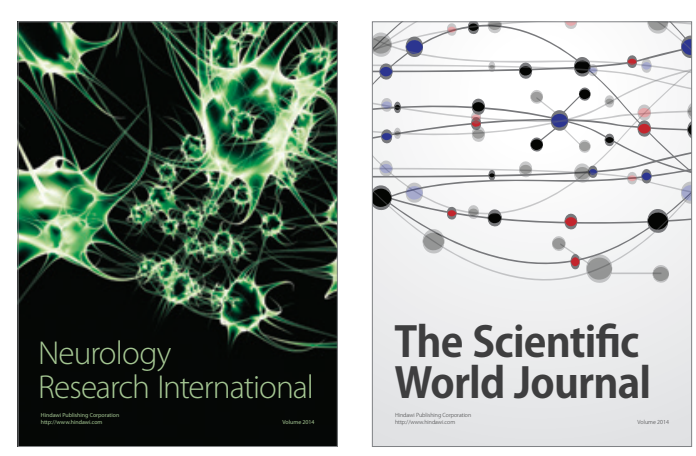

The Scientific World Journal

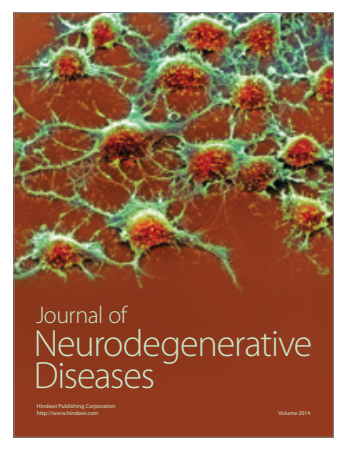

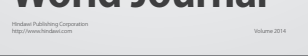

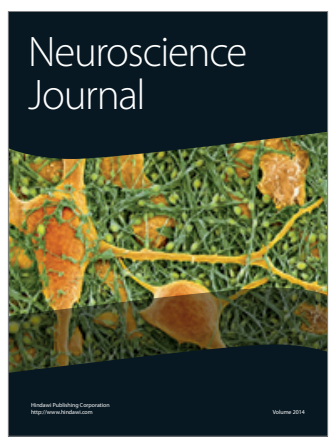

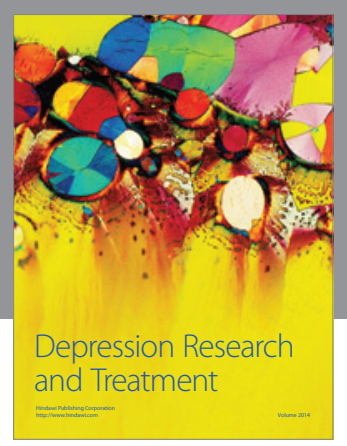
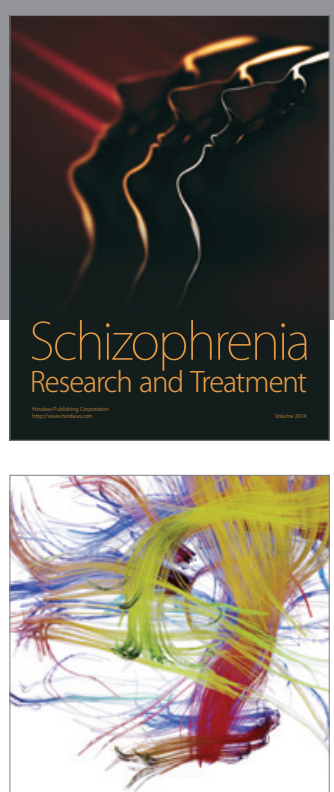

Brain Science

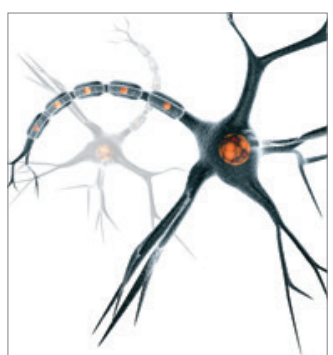

Neural Plasticity
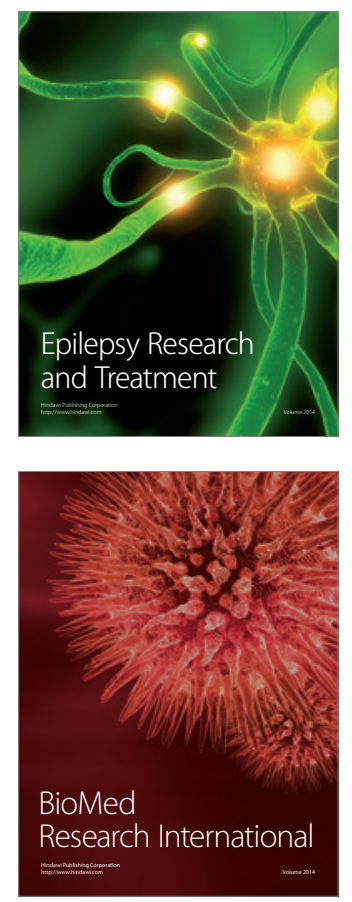

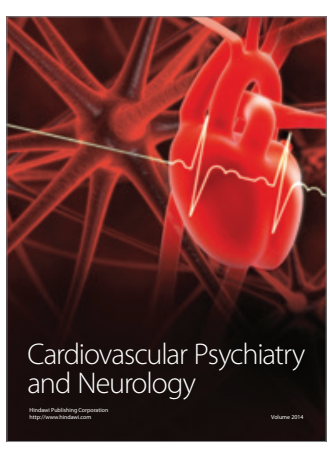

Parkinson's

Disease
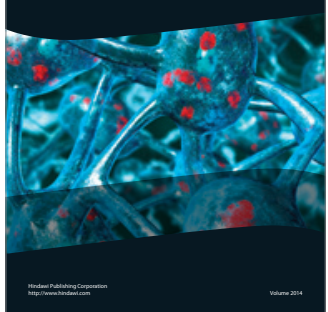